特別講演 II

\title{
LE SYSTEME RETICULO-ENDOTHELIAL EST-IL METABOLIQUEMENT AUTONOME?
}

R. Wegmann

Institut d'Histochimie Médicale, Faculté de Médecine-Paris

\section{Introduction}

Le métabolisme des cellules du système réticulo-histocytaire pose des problèmes sur les fonctions nutritionnelles de ces cellules et surtout de leurs rapports avec les tissus hôtes. Ces cellules exercent des fonctions régulatrices dont il ne faut pas sous-estimer l'importance. En raison de sa diffusion le SRE ne peut être isolé, enlevé ou être analysé pour lui-même. L'un des moyens de mesurer son activité est de le bloquer en supprimant toute activité de ces cellules en l'encombrant de particules inertes. Mais cette méthode a le tort de vouloir démontrer la beauté du corps après l'avoir transformé en momie. Une autre technique d'analyse consiste à les stimuler pour observer le comportement de ces cellules. Dans ces conditions à quels critères recourir pour évaluer la capacité réactionnelle de ces cellules, en admettant que ces cellules possèdent une potentialité qui leur soit propre? Une des méthodes qui semble la plus fructueuse pour examiner ce dernier point consiste à analyser le comportement cytoenzymatique de ces cellules par rapport à leur hôte, tant dans le foie que dans la rate en tâchant d'apprécier les relations réciproques qui s'effectuent entre eux.

\section{Matériel \& Technique}

Des souris mâles inbred, adultes ont reçu, par animal, une unique injection, par voie intraveineuse, à la dose de 400 gamma d'un extrait microbien dénommé Wx B 3148. Les animaux ont ensuite été sacrifiés respectivement après 24 heures, 3 jours, 8 jours, 15 jours, 30 jours et 60 jours. Au moment du sacrifice des animaux témoins ont été utilisés pour servir de comparaison afin de définir à tout moment l'aspect normal par rapport à l'aspect expérimental.

\section{Résultats morphologiques}

Dès le sacrifice, des prélèvements de ces organes ont été, les uns fixés classiquement dans une solution de Bouin et, ou, dans du formol pour l'hématoxyline éosine et le Masson, les autres fixés à l'acétone glacial pour certaines réacttions enzymatiques. Une partie a été congelée à la température de la neige carbonique pour l'étude de quelques systèmes enzymatiques et coupée aussitôt au cryotome. Les enzymes étudiées sur tissu frais sont les suivantes:

- la glucose-1-phosphatase et la glucose-6-phosphatase (Chiquoine, 1953)

- la glucose-6-phosphate deshydrogénase (Wegmann \& Gerzeli, 1961)

- la 3-phosphoglycéraldehyde deshydrogénase (Himmelhoch \& Karnovsky, 1961)

- la lactico-deshydrogénase, la succino-deshydrogénase et la malicodeshy-drogénase

(Wegmann \& Coll,. 1960 et Verne et Coll, 1961) 
Sur tissus fixés à l'acétone ont été étudiées la phosphatase alcaline (selon Takamatsu) et la tweenestérase (selon Gomori). De plus, on a caractérisé le glycogène (selon Mc Manus) et les acides nucléiques (selon Brachet) (le tout: Lison, 1960)

Dans les conditions d'expérience, on n'a pu observer des modifications apparentes du système ganglionnaire. On ne notait aucune hypertrophie visible dans tous les secteurs examinés. Par contre, le foie et la rate ont présenté une nette hypertrophie visible dès le 8ème jour, qui est devenue maxima le 15ème jour. A ce moment, des nodules blanchâtres parsemaient la surface hépatique, alors que la rate restait indemne d'un pareil aspect moduleux.

Toutes ces techniques n'appellent pas de discussion particulière. Notons seulement que nous avons eu recours pour la caractérisation des deshydrogénases du Nitro bleu de tétrazolium, type III de Sigma, à l'exclusion de tous les autres.

\section{Colorations classiques}

Dans le foie :

Dans l'ensemble, par les colorations classiques à l'H.E et au trichrome de Masson, on note quelques infiltrations lymphocytaires après 24 heures, ainsi qu' une mobilisation des cellules de Kupffer dès le 3ème jour. Le remaniement le plus profond du lobule apparaît au 8ème jour. Au milieu d'amas lymphoides et nécrosés un processus déjà intense permet d'affirmer une hépatite congestive associée à une nécrose nodulaire au début. Et c'est au 15ème jour que se constitue l'hépatite nodulaire avec forte infiltration lymphoide et nécrose disséminée. L'espace porte subit un remaniement profond. Après cette période on observe une régression nette de toutes ces lésions qui s'avéraient aussi transitoires. Vers le 30ème jour, l'infiltration nodulaire est des plus réduites, restant localisée à la région périportale, alors que celle centrolobulaire a pratiquement disparue. Au 60ème jour, la régénérescence du tissu hépatique a repris tous ses droits et il ne persiste plus que de très rares petits amas lymphoides.

Dans la rate:

On note une évolution parallèle. Le début de l'infiltration se manifeste dès le 3ème jour accompagnée d'une dilatation des sinus. La pulpe rouge est envahie par de nombreux globules rouges en lyse et les cellules réticulées sont souvent vacuolisées. Le remaniement le plus manifestes opère après huit jours, donc avec un retard de 5-6 jours par rapport au foie. La pulpe rouge est entièrement infiltrée par des amas lymphoides par quelques plasmocytes et par de nombreux mégakaryocytes, associée à une forte èosinophilie tissulaire. La pulpe blanche n'est pratiquement pas modifiée.

L'augmentation des mégakaryocytes atteint son chiffre maxima vers le 15ème jour, puis régresse vers le 30ème jour en même temps que tous ces phénomènes d' infiltration cellulaire rétrocèdent progressivement. Au bout du 2ème mois, les phénomènes de stimulation ont presque tous disparus pendant que la rate a repris ses dimensions et son volume normaux. 


\section{Résultats cytochimiques}

\section{Glycogène.}

Chez les souris témoins au cours de toute la durée de l'expérimentation les variations en glycogène dans les cellules hépatiques n'ont été guère appréciables, subissant à peine quelques fluctuations dues à la quantité de nourriture absorbée. Dans les cellules de Kuppfer on ne note à aucun moment du glycogène optiquement visible.

Chez les animaux traités on observe dans la cellule parenchymateuse une augmentation très sensible du taux de glycogène, tant dans les zones périlobulaires que dans les zones centro ou médiolobulaires. Cette répartition est uniforme dans les cellules. Une digestion par l'amylase montre bien qu'il s'agit de glycogène et non d'autres polysaccharides. Dans les cellules de Kuppfer, le glycogène apparaît discrètement mais ne subit pratiquement pas de fluctuations au cours de toute la durée de l'expérimentation (Fig. 2).

\section{Acides nucléiques}

Les souris normales ne montrent qu'un taux moyen d'acide ribonucléique tant dans leurs cellules parenchymateuses que dans les cellules du SRE (Fig. 1 et 6).

Au cours de l'expérimentation la charge en RNA devient considérable dans les cellules parenchymateuses et dans les cellules du SRE locales ou en mobilisation.

Cette augmentation se traduit très nettement dès la 24ème heure, atteint progressivement des valeurs importantes (Fig. 7) qui est maxima vers le 30ème jour. (Fig. 2, 3, 4, 5 et 7). Après cette période une diminution brutale du taux en RNA se produit, qui tombe loin au dessous de la normale. Ies plus grandes fluctuations se produisent dans la zone centrolobulaire.

Le taux en $A D N$ se modifie aussi mais précède celui de l'ARN, puisque c'est au 15 ème jour que la teneur en ADN paraît la plus importante. Au moment de la charge maxima en ARN (30e jour) le taux en ADN diminue sensiblement.

Cette évolution est parallèle dans les cellules parenchymateuses et du SRE. Il est à noter que les nodules deviennent très riches en ARN, témoignant aussi d'une

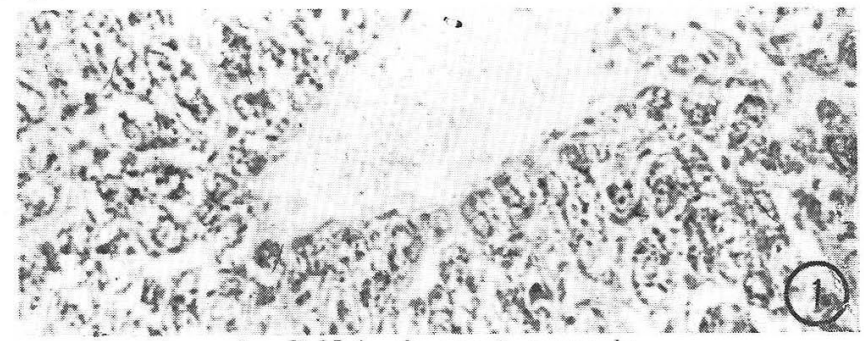

1. R.N.A. de souris normale.

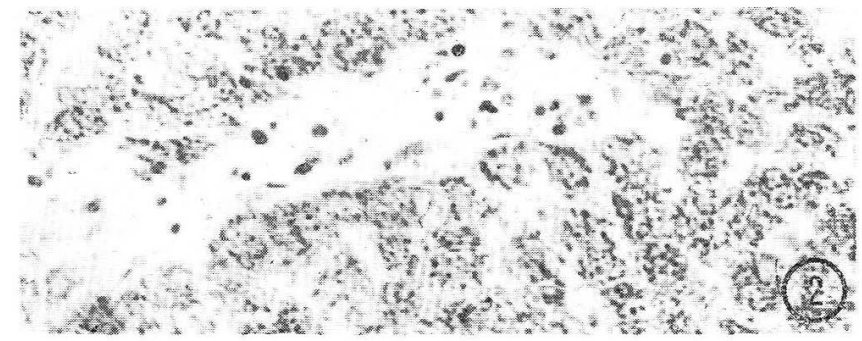

2. R.N.A. après 24 heurs de traitement. 


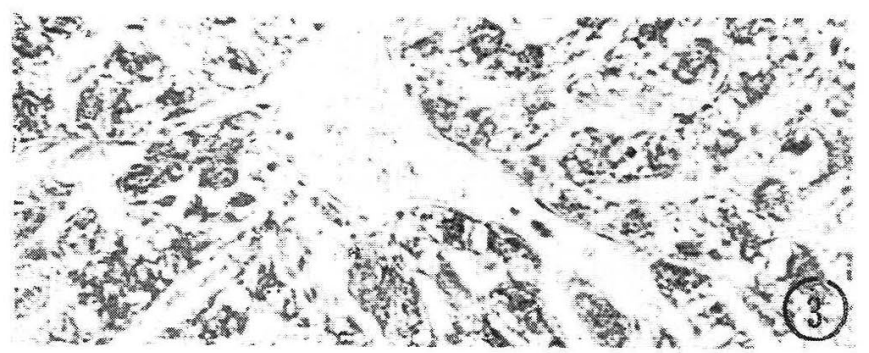

\section{R.N.A. après 3 jours.}

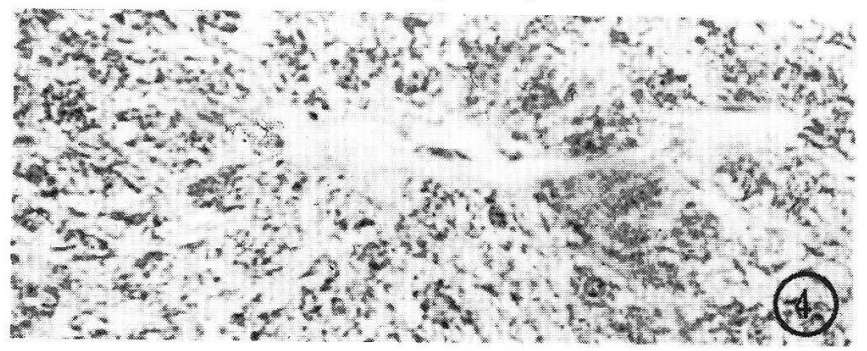

4. P.N.A. après 8 jours.

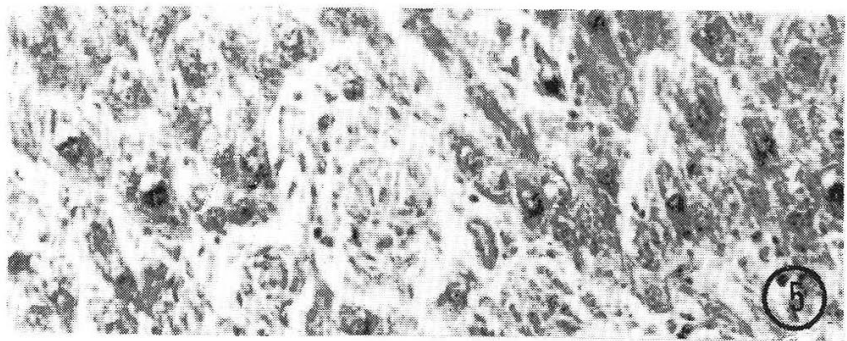

5. R.N.A. après 30 jours L'activitè est notable.

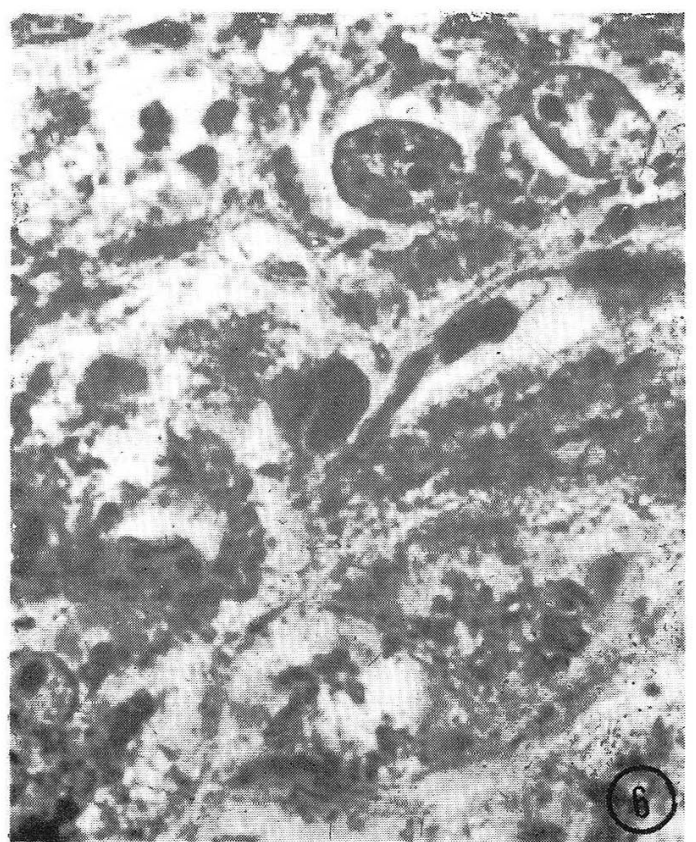

6. R.N.A. chez le témoin: réaction moyenne.

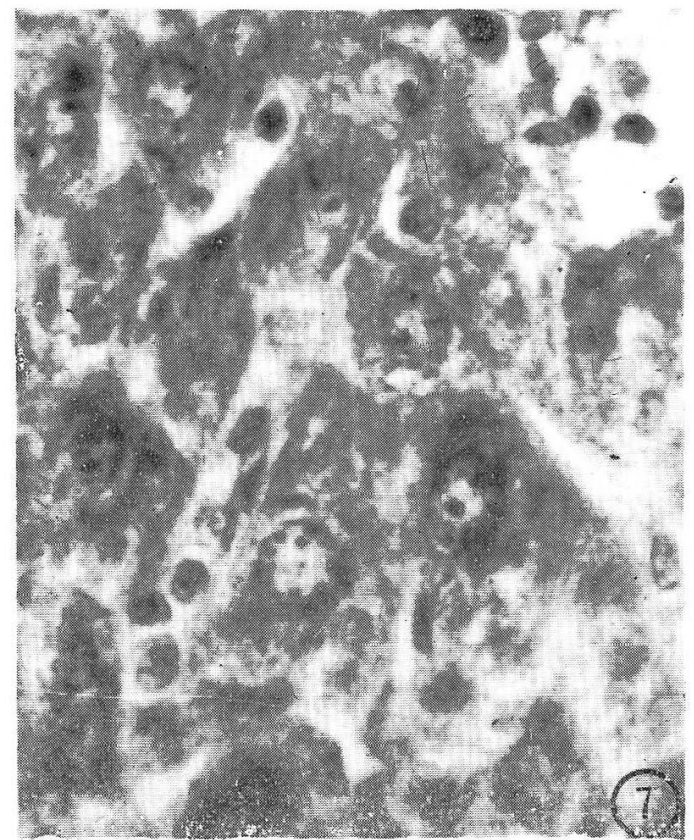

7. R.N.A. après 15 jours: réaction plus forte. 
forte stimulation de leur activité ribosomique.

Les lipides :

Les souris ne possèdent qu'un taux assez faible en lipides visibles dans leurs cellules parenchymateuses et très peu d'enclaves dans les cellules de Küppfer. On note une augmentation passagère de ces enclaves dans toutes les cellules, mais leur localisation dans le lobule ainsi que dans les cellules du SRE est irrégulière. Très souvent les gouttelettes sont libres entre les cellules.

La phosphatase alcaline :

Les animaux normaux ne possèdent pas de phosphatase alcaline, ni dans les cellules parenchymateuses, ni dans les cellules du SRE (Fig. 8). Cette activité apparaît dans les cellules Küppfériennes dès la 24ème heure de stimulation (Fig. 9) puis s'étend progressivement aux cellules parenchymateuses. Quand les nodules intrahépatiques se forment ce sont surtout les cellules périphériques des nodules qui réagissent le plus activement, contemporaines de la formation des nodules et disparaissant avec elles. Le maximum de réaction se situe le 15ème jour après l'injection puis diminue et disparaît rapidement.
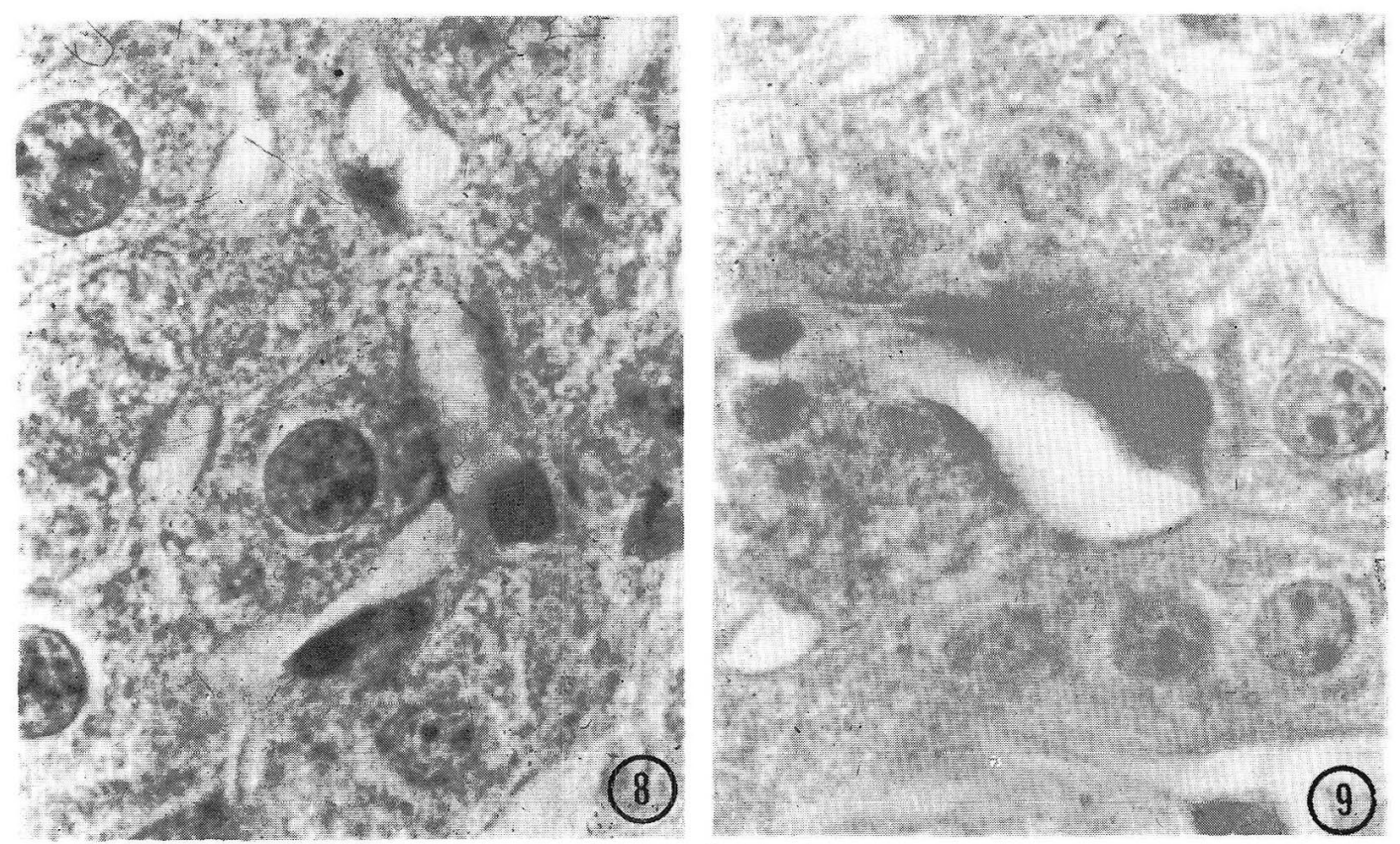

8. a GP-ase alcaline: pas de réaction chez le témoin.

9. $a$ GP-ase alcaline: apposition dans la cellule de Kuppfer après 24 heurs de traitement.

\section{Les estérases :}

L'activité estérasique des souris témoins est importante dans les cellules parenchymateuses et peu intense dans les cellules de Kuppfer (Fig. 10et 12). Très rapidement après l'administration de l'extrait bactérien on assiste à une dégranulation cytoplasmique avec une répartition différente des estérases plutôt qu à une diminution (Fig. 11) dans les cellules des deux types. Puis progressivement, 
quand se forment les nodules, l'estérase augmente d'intensité dans les cellules parenchymateuses jusqu'à atteindre un taux très important réellement supérieur à tous ceux observés jusqu'alors. Par contre, dans les cellules mobilisées du SRE ainsi que dans les nodules l'activité enzymatique reste très faible ou muette. On observe (Fig. 13) des cellules parenchymateuses qui, semblant se transformer en des cellules d'un autre type, paraissent s'intégrer - dans les nodules histocytaires. Quand les nodules régressent, la charge estérasique reste faible autour du nodule en même temps que s'observe le même phénomène de dégranulation des cellules parenchymateuses près du centrolobule (Fig. 14). Cet aspect visible au 30ème jour s'accentue au 60ème jour, date à laquelle le périlobule ne contient que très peu d'estérases et que le centrolobule en est relativement dépourvu. Cet aspect peut étre suivi encore sur les figures, 15 à 17.
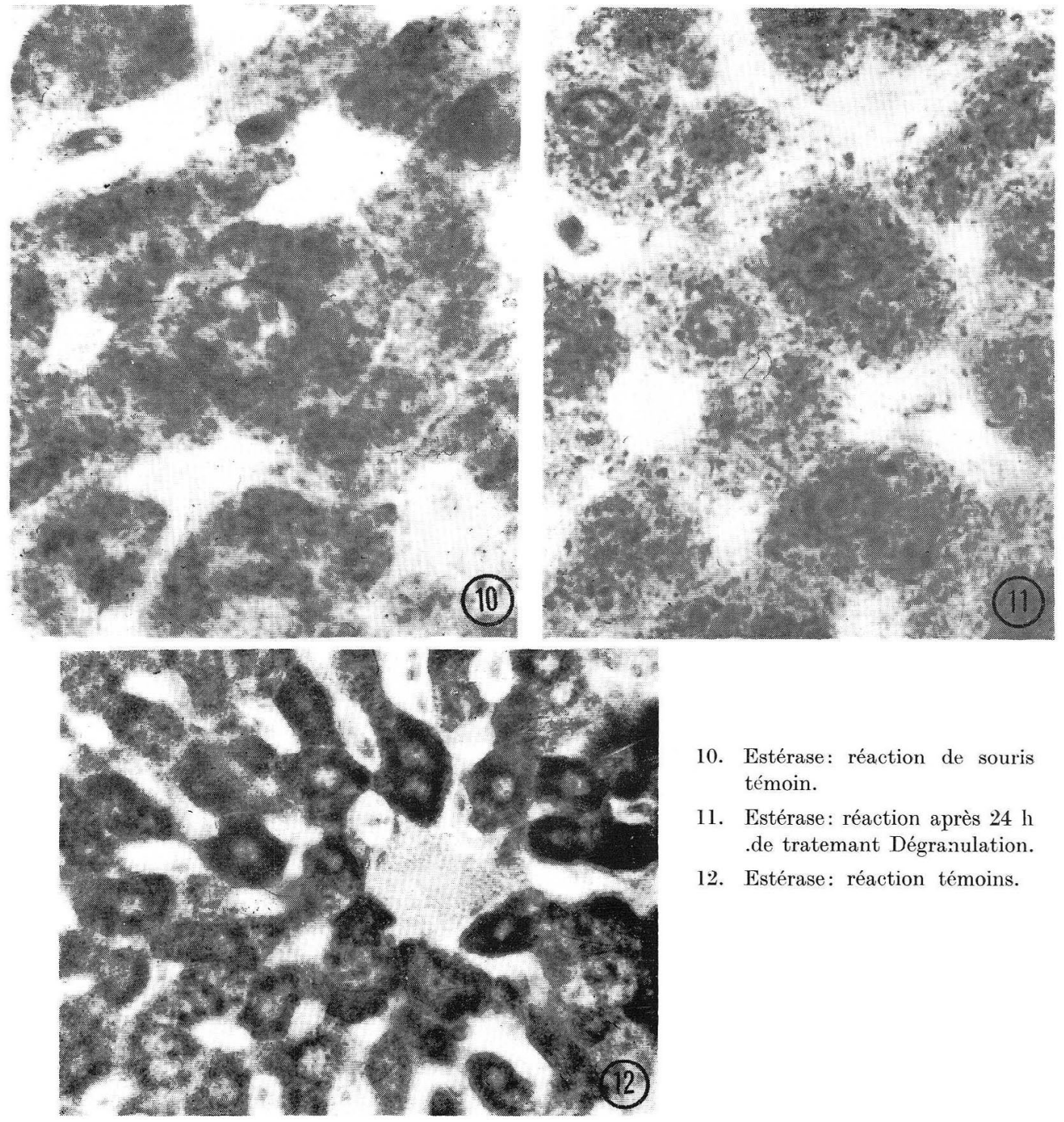

10. Estérase: réaction de souris témoin.

11. Estérase: réaction après $24 \mathrm{~h}$ .de tratemant Dégranulation.

12. Estérase: réaction témoins. 


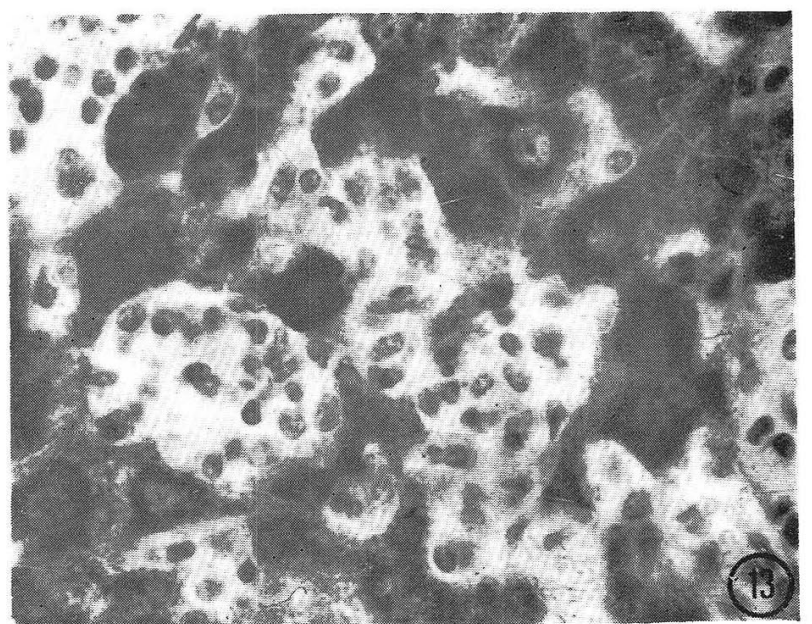

13. Estérase: après $15 \mathrm{j}$. de traitement. Nodules sans activités et cellules parenchymateurs très ${ }^{*}$ actives.

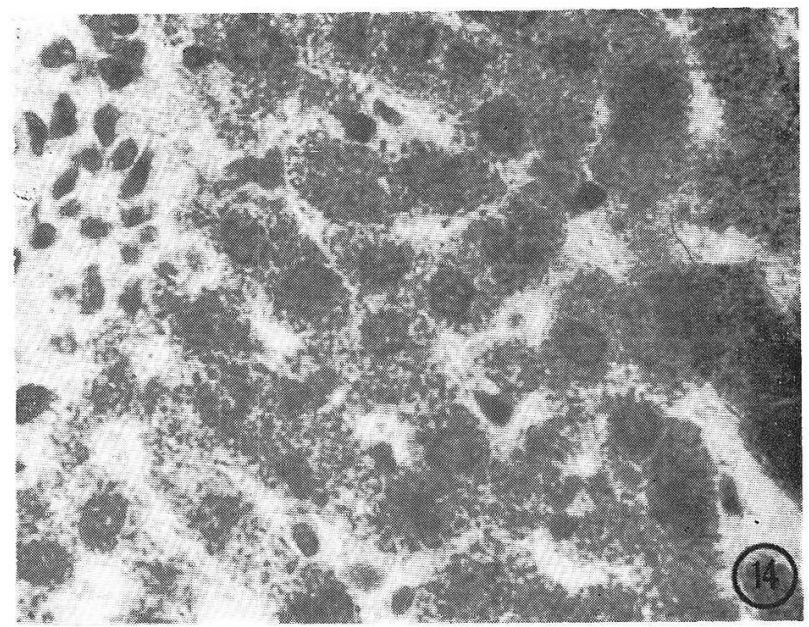

14. Estérase: après 30 j.: dégranulation.

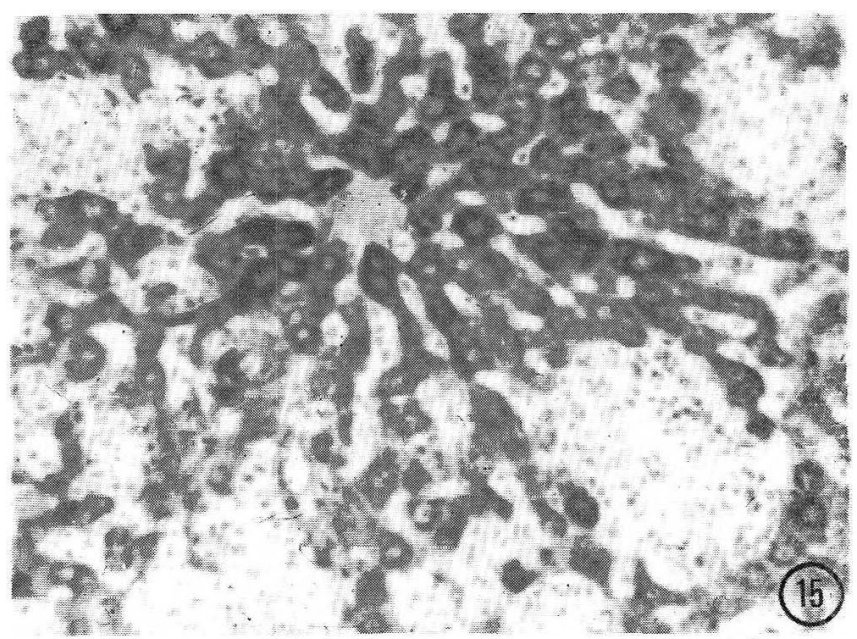

15. Estérase: après 15j.: vue générale avec nodules. 


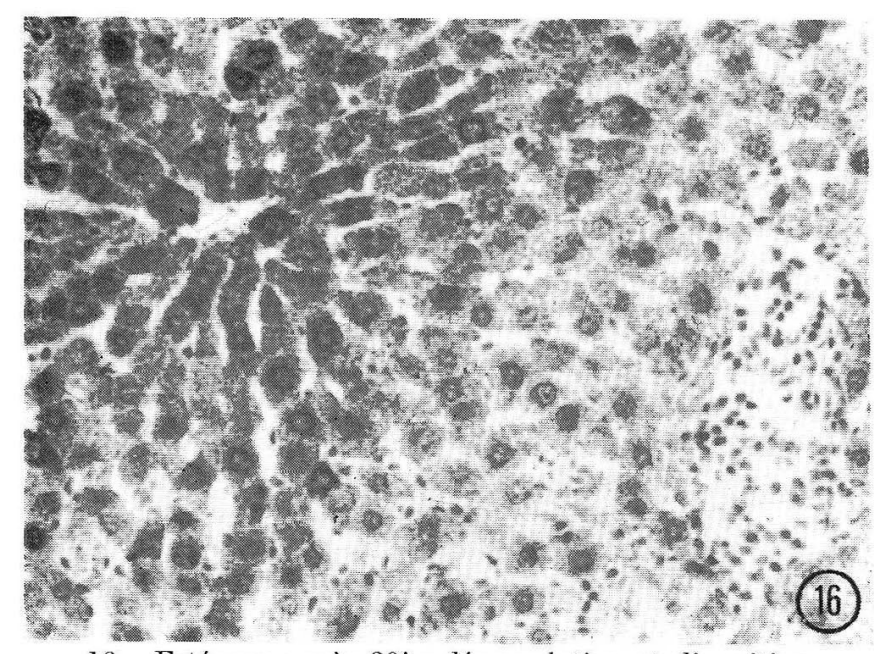

16. Estérase: après $30 \mathrm{j}$.: dégranulation et dinunitins

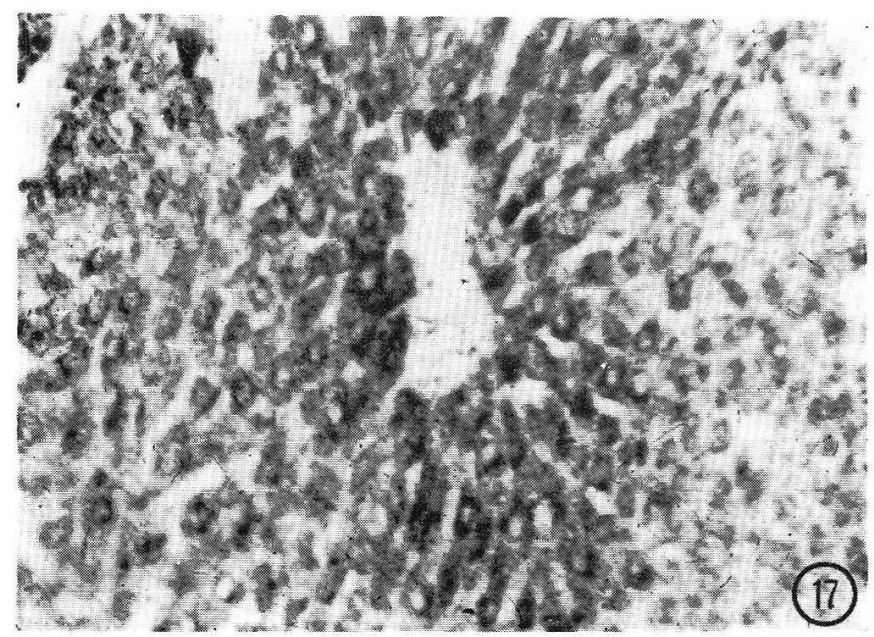

17. Estérase: après $60 \mathrm{j}$.: Localisation centrolobulaise seule.

\section{La glucose-6-phosphatase :}

Elle est assez active chez les souris témoins, plus intense autour du centrolobule que du périlobule (Fig. 18). On ne distingue aucune trace de cette enzyme dans les cellules de Kuppfer ou dans les cellules en voie de mobilisation. Mais aussitôt après l'injection on assiste à une chute remarquable de la glucose-6phosphatase dans tous les éléments cellulaires, aspect qui persiste pendant toute la durée de l'expérience, comme on peut le voir au 15ème jour très nettement (Fig. 19).

\section{La glucose-6-phosphate deshydrogenase :}

Elle est relativement active dans le parenchyme de foie de souris normale (Fig. 20), surtout dans l'espace périportal et peu active dans le centrolobule. Les cellules du SRE sont inactives, sous l'influence du traitement stimulateur en 8 à 15 


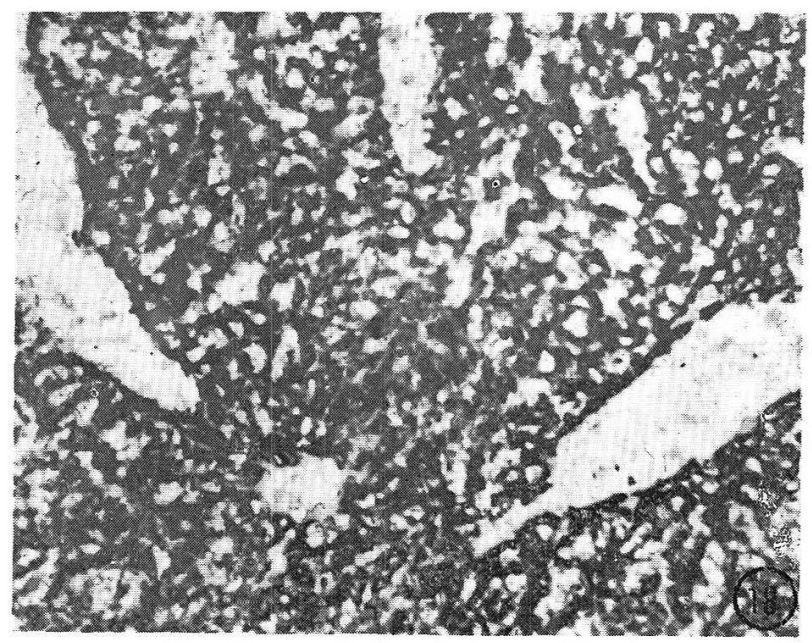

18. Glucose-6-phosphatase. Témoin.
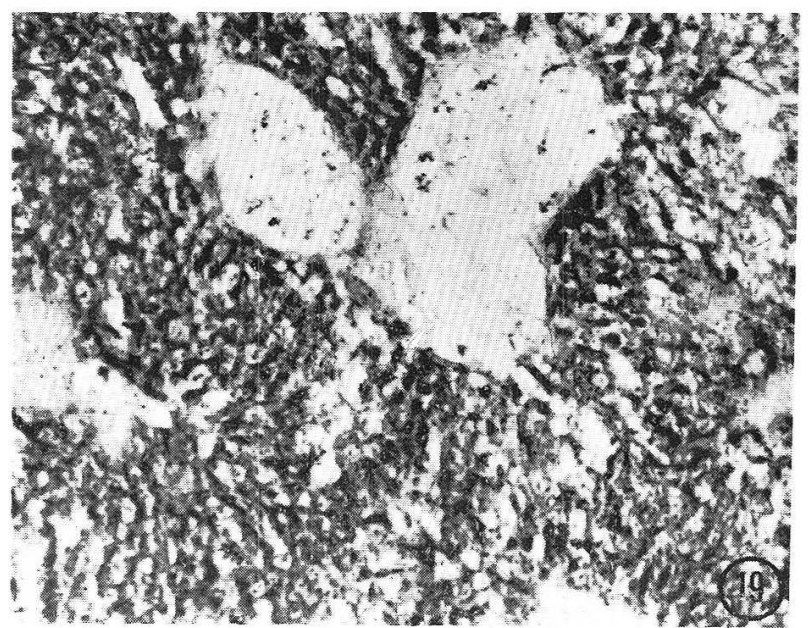

19. ido: cprès 8 jours

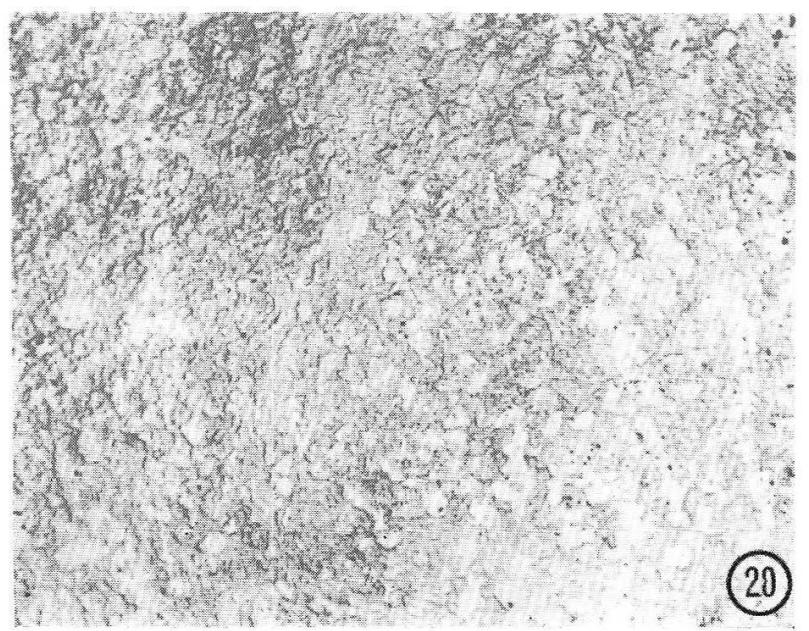

20. ido: après 15 jours. 


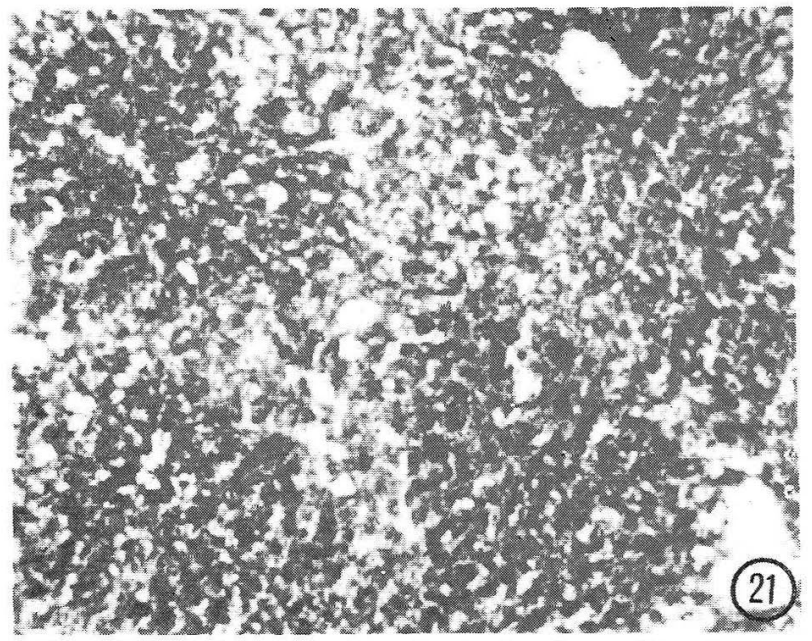

21. Glucose-6-phosphrtase doz hydøogénase Témoine.

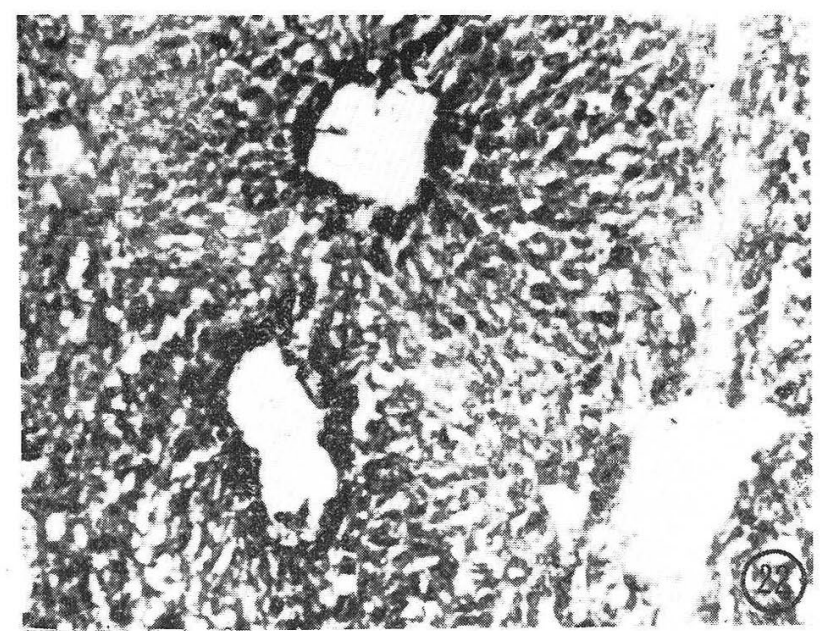

22. ido. après 8 jours de traitement.

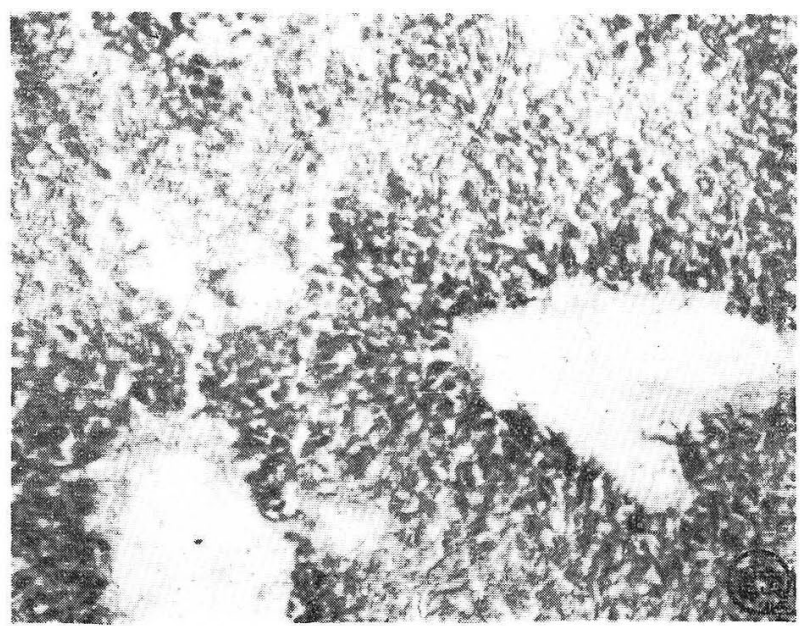

23. ido après 15 jours de traitement. 
jours l'espace périportal est nettement activé(Fig.21)mais le reste n'est guère modifié, sauf le centrolobule où les cellules parenchymateuses paraissent moins actives. Cet aspect cependant ne se maintient pas. Après cette période, on assiste à une diminution de ces activités, la chute la plus forte se produisant dans les cellules parenchymateuses du périlobule (Fig. 22). Plus tard, l'aspect redevient progressivement normal.

\section{La 3-phosphoglycéraldéhyde deshydrogénase :}

Les variations que subit cette enzyme sont très voisines de l'enzyme G6P-DH et l'on peut prendre la description antérieure comme base de référence, bien que le système enzymatique soit intégré à tout un autre niveau que le précédent.

\section{Les autres systèmes enzymatiques :}

Il est intéressant de signaler que ni la succino, ni la lactico, ni la malicodeshydrogenase ne montrent de variations susceptibles d'être retenues tant chez l'animal normal qu'au cours de ces jours d'expérience.

\section{Dans la rate:}

Si nous tenons compte des faits précédents, il est très difficile de les mettre aussi clairement en évidence dans la rate, par suite des diversités des types cellulaires et $\mathrm{du}$ peu de distinction possible des réactions enzymatiques.

Il ne nous a pas été possible de suivre clairement les variations d'activité sauf au niveau des mégakaryocytes mais nous ne pouvons les considérer dans cette interprétation que pour une phase déterminée et non pendant toute la durée de la stimulation. La seule impression générale à retenir est que les variations observées sont plus faibles et plus tardives que dans le foie. Mais on ne peut indiquer rien de précis en ce qui concerne les cellules réticulaires.

\section{Discussion}

Le problème que nous nous étions posés était le suivant: qui est-ce qui supporte l'activité métabolique du système réticulo-histocytaire? La réponse n'est certes pas aisée, mais elle pourrait recevoir un début de solution. Nous remarquons tout d'abord que le glycogène absent dans les cellules réticulaires du témoin apparaît au cours de l'expérimentation: c'est la seule manifestation d'un stockage métabolique et que les estérases ne se manifestent qu'au bout d'un délai assez long, environ 8 jours après l'injection de l'extrait bacillaire.

Au contraire, nous remarquons de très vives modifications tant du stock en glycogène que des acides nucléiques du parenchyme, associées à des variations sensibles des enzymes. La chute de glucose-6-phosphatase démontre que le foie ne libère pratiquement plus de glucose en excès dans le torrent circulatoire. Le cycle de Krebs en même temps est bloqué. Les processus régulateurs de la cellule parenchymateuse cessent en même temps que le taux des hydrolases augmente (phosphatase alcaline). Cette période d'attente dure environ 8 à 10 jours. Puis on constate une élévation des estérases considérable suivie de très près par une augmentation de l'acide ribonucléique. On remarquera que l'activité glucose-6-phosphate deshydrogénasique intervenant dans la formation de NADPH nécessaire à 
la biosynthèse des lipides, mais formant aussi du ribose-5-phosphate dans la voie des pentoses est légèrement stimulée avant que les estérases et la ARN augmentent. Il semble donc bien y avior une corrélation. Or, à quel moment observons-nous la plus forte activité phosphatasique alcaline dans les cellules de Kuppfer? au 15 ême jour! moment où les processus anaboliques et cataboliques s'équilibrent dans', ou, au voisinage des nodules formés (TAb I et II, avec peur de détails).

De pareilles modifications se produisent sans aucun doute par la nature du produit injecté. Il est à supposer que le stimulus provient de l'extrait bacillaire et que ce dernier résorbe à partir de la lumière de la veine porte, passe par les sinusoides. Cette phase est contemporaine de la chute de la plupart des activités observées, qui vraisemblablement passent à travers les cellules de Kuppfer. Il résulte ensuite une stimulation des cellules parenchymateuses secondaire qui permet aux cellules Kuppfériennes de se développer, nous allions dire de "s'implanter" dans les sinusoides pour former les nodules. Cette stimulation se produit

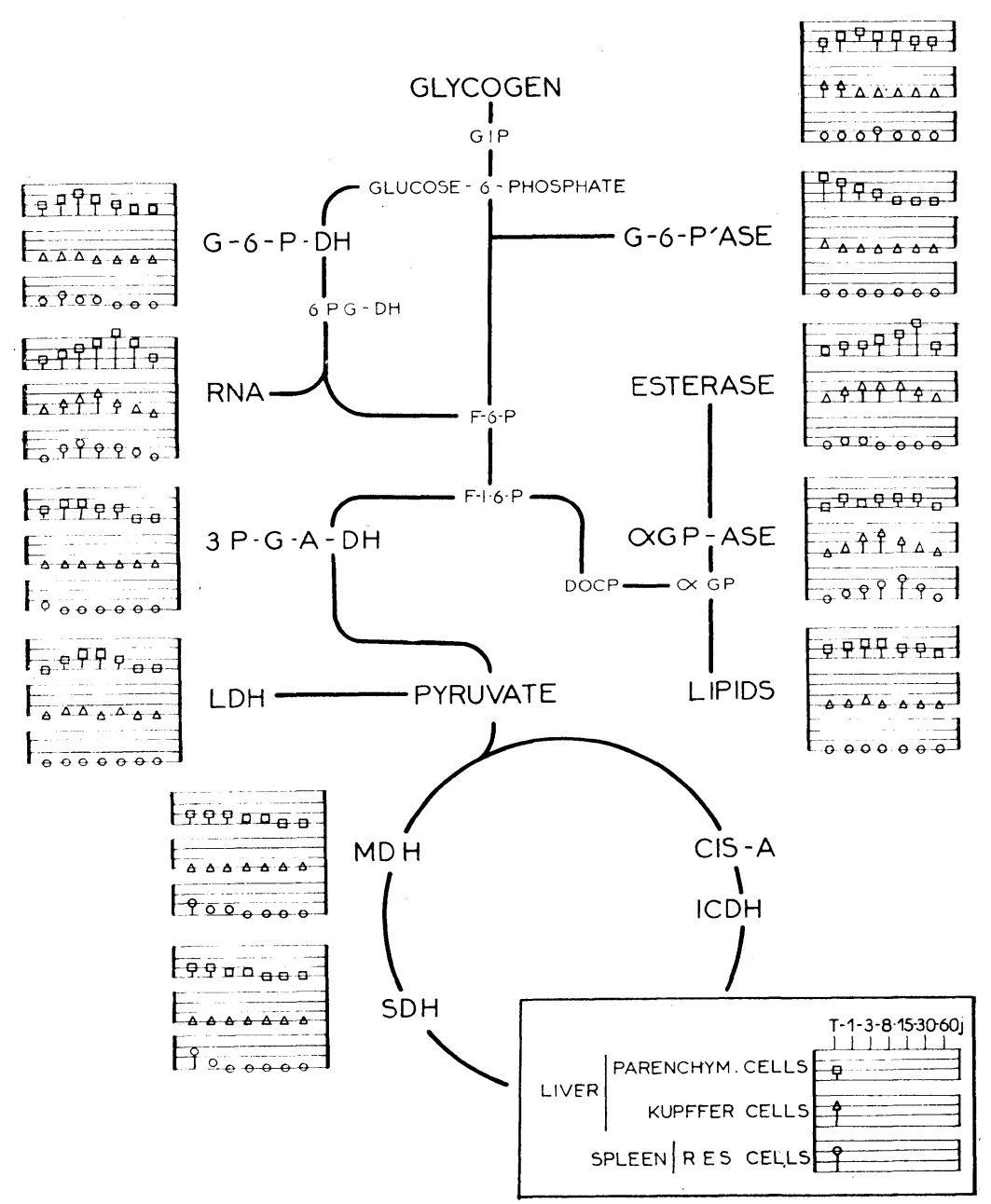




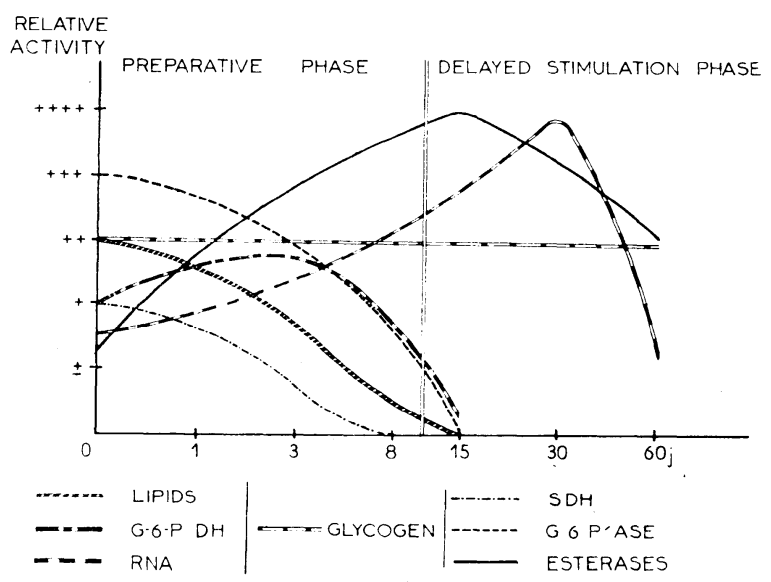

donc par un effect indirect sur le parenchyme. Comme l'ont montré Halpern, Haguenau et Hollmann (1963), on ne note guère dans ces cellules au réticulum endoplasmique ou d'ergastroplasme ou encore la plupart des organitès et structures visibles dans la cellule parenchymateuse. On peut donc admettre que la fonction de la cellule SRE est totalement différente de la première et que si elle possède des fonctions particulières elle ne peut les effectuer que grâce au support dont elle bénéficient. On sait d'après les travaux de Berliner et Dougherhy (1960) que la cellule RES retient et métabolise les stéroides. Si l'on tient compte du fait que le pourcentage des cellules parenchymateuses du foie de souris est de: $60.6 \%$, celui des cellules RES de: 33.4\% (Daoust, 1958); Berliner et Dougherhy ont montré que seuls les hépatocytes conjuguent les stéroides en quantités significatives, alors que les deux types de cellules sont capables de réduire et d'oxyder le noyau stéroide.

Il existe donc des différences manifestes entre la cellule parenchymateuse et la cellule de SRE. Nous avons pu montrer que les propriétés de phagocytose ne dépendent nullement de leurs activités enzymatiques, relativement réduites, mais semblent plutôt dépendre de facteurs externes à eux. On pourrait prendre comme terme de comparaison l'acitivité d'un porte avions que serait le foie et dont les avions seraient les cellules du SRE et dont la bonne marche dépendrait du degré de ravitaillement du bateau. Ceci ne veut nullement dire que les cellules du SRE soient métaboliquement inactives; du moins in vivo elles se comportent comme si elles devaient compter sur une base de ravitaillement. Il' n'a été tenu compte que d'une dose dans cette expérience et on n'a point eu recours à un blocage préalable par des colloides du type carbone, phosphate de chrome ou d'albumine iodée dénaturée d'après les conditions élaborées par Benacerraf et Coll., (1957). Mais il est à peu près sûr que les lipides en émulsion sont assimilés d'abord par les cellules parenchymateuses. Par l'iode ${ }^{131}$ oléine ce taux était de $49 \%$ dans ces dernières et de $2 \%$ dans les cellules Kuppériennes. On peut donc admettre que le métabolisme des cellules du SRE n'atteint le plein développement de leur activité que lorsque les cellules parenchymateuses ont elles-mêmes contribué largement à leur métabolisme. 
Pourquoi, dans ces conditions, les cellules du SRE de la rate augmentent-elles le nombre des mégakaryocytes? On sait leur rôle dans la formation des plaquettes mais on ne sait pas pourquoi ce nombre de cellules se multiplie. Qu'il y ait une intervention dans la crase sanguine cela est certain mais on ne pourrait en tirer des conclusions quant au rôle métabolique (Fereira., 1961). Il n'est certainement pas d'order lipidique, comme c'est le cas des cellules du SRE du foie (Di Luzino., 1960), car les modalités réactionnelles du SRE peuvent être très diverses suivant le type de stimulation (Baillif., 1960), et suivant la méthode de stimulation (Snell., 1960.)

\section{En conclusion}

Nous admettons que le rôle du parenchyme dans le "ravitaillement" des cellules du SRE est capital et que ces dernières sont en quelque sorte tributaires des premières, sans lesquelles elles ne parviennent pas à leur plein développement et à leur pleine activité. Leur équipement enzymatique est en effet trop faible pour assumer seules toutes ces activités multiples. Nous considérons que ce rôle peut varier en fonction des lieux d'activité. Ceci nous est indiqué par la récation différente observée dans le foie et dans la rate.

\section{RÉSUME}

L'injection d'un extrait bactérien Wx B 3148 provoque une forte modification des estérases et de la glucose-6-phosphatase du parenchyme hépatique ainsi que des acides nucléiques. Lorsque cette stimulation est maxima, on observe alors des modifications du SRE, du type prolifératif. Les autres activités:

- phosphatasique alcaline, glucose-6-phosphatase-DH et 3-PGADH jouent un rôle moindre

- La SDH, la MDH et la G-6-P-DH n'interviennent pas

- Le SRE n'est actif que si son parenchyme de support l'est, ou le devient. 


\section{Litlérature}

1) Baillif R.N.

Reaction patterns of the reticuloendothelial system under stimulation Ann. N.Y. Ac. Sci., 1960, 88: 3-13.

2) Benacerraf B., Biozzi G., Halpern B.N. et C. Stiffel

Physiology of phagocytosis of particles by the RES. In physiopathology of the reticulo-endothélial system:

52 B.N. Halpern Ed. Blackwell Scientific Publ. Oxford., England

3) Berliner D.L. and Dougherty

Influence of reticulo-endothelial and other cells on the metabolic fate of steroids Ann. N.Y. Ac. Sci.. 88: 14-29, 1960.

4) Chiquoine D.A.

The distribution of glucose-6-phosphatase in the liver and kidney of the mouse J. Histochem. Cytochem., 1953, 1 : 429-435

5) Daoust R.

The cell population of liver tissue and the cytological reference base in liver function: $3-10$

R.W. Brauer ed. Am. Inst. Biol. Sci. Washington D.C.

6) Di Luzino N.R.

Reticulo-endothelial involvment in lipid metabolism Ann. N.Y. Ac. Sci., 88: 244-251, 1960.

7) Ferreira David

Sur la structure et lepouvoir phagocytaire des plaquettes sanguines $Z$. Zellforsch., 1961, 55: 89-103

8) Halpern B.N., Haguenau F. et Hollmann K.H.

Modifications ultrastructurales observées dans le foie et dans la rate de souris après injection d'extraits bactériens. Rôle du système réticulo-endothélial dans l'immunité antibactérienne et antitumorale

Coll. Int. CNRS N 115, CNRS édit. Paris., 249-261, 1963.

9) Himmelhoch S.R. et Karnovsky M.J.

The histochemical demonstration of glyceraldehyde"-3-phosphate dehydrogenase activity.

10) Lison L.

J. Biophys. Biochem. Cytol., 9: 573-581, 1961.

11) Snell J.F.

Histochimie animale (2 volumes)-Gauthier-Villars, édit.-Paris., 1960

The reticulo-endothelial system. I. Chemical methods of stimulation of the reticulo-endothelial system Ann. N.Y. Ac. Sci., 88: 56-77, 1960.

12) Wegmann R. et Tordet-Caridroit C.

Histochemical demonstration of succinic dehydrogenase by specific inhibition and activation J. Histochem. Cytochem., 8: 348-349, 1960.

13) Wegmann R. et Gerzeli G.

La glucose-6-phosphate deshydrogénase et ses corrélations avec des substrats voisins du glucose-6-phosphate. Ann. Histochim., 6: 111-124, 1961.

14) Wegmann R., Verne J., Hebert S. et Tordet-Caridroit C.

La mise en évidence de l'acide lactique et de la lacticodeshydrogénase dans les cultures de tissus Ann. Histochim., 5: 41-45,1961.

\section{討}

Seno. S. (Okayama Univ Dept of Path) :

Did you find any relation between the func- tions of reticuloendothelial cells and the adjacent cells in spleen or lymphnode? 